\title{
Effect of Body Wash with Tea Tree Oil on the Prevention of Methicillin-Resistant Staphylococcus aureus in Critically Ill Patients at a university hospital in Egypt
}

\author{
Nahla Shaaban Khalil ${ }^{1 *}$, Asmaa Ibrahim Abo Dead's², and Rahma Mousa Abdelazeem ${ }^{3}$ \\ ${ }^{1}$ Assist professor at Critical Care and Emergency Nursing, Cairo University, Egypt \\ ${ }^{2}$ Lecturer at Critical Care and Emergency Nursing, Mansoura University, Egypt \\ ${ }^{3}$ Registered nurse at Mansoura University Hospital, Egypt
}

*Corresponding author: Nahla Shaaban Khalil, Assist professor at Critical Care and Emergency Nursing, Cairo University, Egypt.
Received Date: April 01, 2019

Published Date: April 24, 2019

\begin{abstract}
Background: Colonization with Methicillin-Resistant Staphylococcus Aureus (MRSA) is independently is associated with mortality in critically ill adults. Tea tree has broad bactericidal activity. Clinical evidence supports its efficacy in eradicating MRSA, but there are no published data on its role in preventing MRSA colonization.
\end{abstract}

The aim of the study: To determine whether the daily use of 5\% tea tree oil compared with standard care (soap and water) had a lower incidence of Methicillin-Resistant Staphylococcus Aureus colonization.

Material and Methods: A quasi-experimental design was utilized and conducted at the neurological, intensive care units at Mansoura University Hospital including 120 adult critically ill patients participated in the study.

Results: There were highly statistically significant differences between the study and control groups on the seventh day. So, all the study group subjects' swabs from nose and groin were free from MRSA, while all the control group subjects' swabs showed positive for MRSA infection.

Conclusion and recommendations: Tea tree oil is effective in preventing MRSA infection. Therefore, the establishment of using tea tree oil in the prevention of MRSA infection and further researches are highly recommended on a larger probability sample in the different geographical hospitals in Egypt to ensure generalization of findings.

Keywords: Tea tree oil; Critically Ill patients; MRSA

\section{Introduction}

MRSA is a major nosocomial pathogen that causes severe morbidity and mortality rate in many hospitalized patients especially critically ill patients and considered one of the main causes of the death worldwide [1]. It is the leading cause of life threating problems such as bloodstream infection, pneumonia, surgical site infection, arthritis, osteomyelitis, and endocarditis if not treated quickly; MRSA can cause sepsis and death [2,3]. Moreover, MRSA infection leads to increased hospitalization length and health care costs [4]. Its prevalence in Egypt is high compared to other African countries. Its prevalence of HA-MRSA increased from $48.5 \%$ in 2005 to $69.1 \%$ in 2013 and CA-MRSA $23.3 \%$ to $60 \%$ [5]. Furthermore, about two billion people worldwide carry Staphylococcus aureus, between 2 million and 53 million people in the United States (USA) carry MRSA [6]. Mortality rate due to MRSA is $50 \%$ and higher for patients infected with MRSA in intensive care unit [7]. According to centers for disease control and Prevention (CDC) approximately 2.3 million individuals in the USA diagnosed with MRSA, approximately $86 \%$ of persons diagnosed with MRSA 
associated nosocomial or health care infection and $14 \%$ were community infection, 94,000 cases are MRSA infection and 11,000 dies from MRSA infection in the USA each year [8]. Approximately 25-35\% of the populations carry Staphylococcus aureus bacteria on the skin or nose is generally harmless, the bacteria enter the body through a cut of the skin or another open wound [9]. MRSA infection symptoms generally begin as swollen, painful red bumps that may resemble pimples or spider bites. In addition, it has a wide range of symptoms depending on the infected part of the body. The affected area may be redness, warm to touch, abscess and fever [10]. Critically ill patients usually are exposed to a number of intrinsic and extrinsic factors in ICU increased risk of MRSA. Typically, the patients have multiple invasive procedures or devices as intravenous tubing, hemodialysis catheters, urinary catheters, mechanical ventilation and tracheotomy that may contribute to limitation of patients positioning and mobility, even so, increasing the risk of MRSA occurrence [11]. Moreover, length of ICU stay, antibiotic use, diminished immune response, old age, superficial wound, MRSA colonization adds to comorbidity as diabetes and chronic disease side by side with the patient underlying disease contribute to MRSA development [12]. Additionally, it has been noted that health care workers have an increased risk of colonization and contribute to the transmission of MRSA infections in hospitals [13]. Today, MRSA is not a response to antibiotics therapy; it becomes resistant to beta-lactam antibiotics and causes serious complications [14]. In recent years, natural products such as herbal medicine and essential oil spread increasingly owning to plant naturally derived oil like tea tree oil (TTO). it's a safe and effective herbal source of therapeutic help in the health care system all over the world [15]. TTO has antimicrobial, antifungal and antiviral, and anti-inflammatory properties due to the presence of a compound that is known terpinene- $4[16,17]$. In addition, TTO is safe and well tolerated by patients, and considered as an alternative therapy to prevent and treat MRSA infection [18].

Complementary therapy must be integrated into nursing practice to provide a high standard of holistic care and positive outcomes for the patients, their basic philosophies have been a part of the nursing profession since its beginnings .Critical care nurses should be aware of the legal and ethical obligation related to the use of that herbal therapy in order to promote a healing environment [19].

\section{Subjects and Methods}

A quasi-experimental research design was utilized consisting of a convenience sample of 120 adults newly admitted male and female patients at neurological intensive care units at Mansoura University Hospitals. The inclusion criteria included patients in the age group ranged from 20 to 60 years old. The exclusion criteria included all patients that have known to be colonized, have a positive screening test for MRSA and unlikely to stay in selected ICU. Later, official permission was obtained from the research ethical committee at Faculty of Nursing, Mansoura University as well as, an official letter was obtained from the hospital administrative authority to initiate data collection from assigned patients. Next, patients were interviewed and reported that participation in this study was voluntary and written consents were taken from those patients and relatives. During which the MRSA baseline assessment was carried out; it consisted of patients' demographic data, health relevant data, and MRSA screening. MRSA screening tool was adopted from [20]. It covered laboratory data regarding detection of MRSA by obtaining swabs from nose and groin on admission from ICU patients in both study and control groups. Also, swabs were obtained from the nose and groin on the seventh day after implementation of interventions for both groups that included body bath with tea tree oil( study group)and traditional body wash with soap and water (control group). The MRSA screening tool was reliable utilizing inter-rater reliability with kappa coefficient $=0.92$.

\section{Procedure of data collection}

This study was conducted in three phases; preparation, implementation and evaluation phases.

Preparation phase: This phase involved the preparation of data collection tools. The baseline data were collected from the patient's record, which included data, related to demographic characteristics, health relevant data on admission. It took around 10-15 minutes to be completed for each patient then, it was ended by conduction of the pilot study.

Preparation of Tea tree oil: Tea tree oil5 \% was pure tea tree oil without any addition of other chemicals and made in Pakistan with a trade name Hemani. It was prepared according to the reference recommended dose: add $5 \mathrm{ml}$ of oil into $100 \mathrm{ml}$ of water. The total amount used during body wash was $40 \mathrm{ml}$ of oil into 800 $\mathrm{ml}$ of warm water and mixed [21].

Implementation phase: It was initiated by random assignment of patients to determine the study and control group. The odd number in the patient list was assigned to the control group and the even number to the study group. The researcher obtained swabs from the nose and the groin on admission for both groups before intervention and got the results regarding the detection of MRSA. After completion of the initial screening for MRSA, the results illustrated whether the patient was eligible for participation or not in the current study and till the seventh day.

During this phase, the study group of admission was received a body wash with TTO 5\% once/day and was applied every day utilizing aseptic technique by using a piece of gauze during body wash and not using cotton. So, the cotton absorbs TTO. The lotion of TTO is left on patients' skin till the seventh day for the study group. While the control group of patients was received traditional body wash every day with liquid soap and water once a day. Both groups were exposed to the session of body wash in a time ranged between 30-45 minutes at the morning shift till the seventh day Finally, Swabs were obtained from the nose and the groin of all patients in both group) at the seventh day of intervention.

Evaluation phase: The laboratory swabs results that were obtained on the first and seventh day for both groups were documented and be compared for detection of MRSA. 


\section{Data Analysis}

Statically package for Social Sciences version 16 (SPSS, Inc., Chicago, IL, USA).0.05 level was used as the cut off value for statistical significance and the following statistical measure was used. Chi-square test was used for comparison for qualitative variables. T-test and means \pm standard deviations were used for quantitative variables.

\section{Results}

\section{Patients' demographic characteristics and health relevant data:}

As can be seen from the Table 1 that half and more than two thirds (50\% \& 70\%) of both the study and control groups respectively, their age ranged between $50-60$ years with the mean age $44.65 \pm 11.73$ and $50.12 \pm 10.03$ years respectively. More than two thirds $(65 \%)$ of the study group was female, while two-thirds of the control group was male. Regarding the education level, nearly half of both groups were divided between secondary and primary school graduates. However, no significant statistical differences were found between both groups regarding socio-demographic data except gender $\left(\mathrm{X}^{2}=7.519\right)$.

Table 1: Frequency and Percentage Distribution of patients demographic Data among The study \&The Control Groups $(n=120)$.

\begin{tabular}{|c|c|c|c|c|c|}
\hline \multirow[t]{2}{*}{ Variables } & Study Group & & $\begin{array}{c}\text { Control } \\
\text { Group }\end{array}$ & & \multirow{2}{*}{$\begin{array}{c}\text { Significance } \\
\text { Test }\end{array}$} \\
\hline & $N(60)$ & $\%$ & $N(60)$ & $\%$ & \\
\hline Age & \multirow{2}{*}{9} & \multirow{2}{*}{15} & \multirow{2}{*}{4} & \multirow{2}{*}{6.7} & \multirow{5}{*}{$\chi^{2}=5.323$} \\
\hline $20-29$ & & & & & \\
\hline $30-39$ & 6 & 10 & 4 & 6.7 & \\
\hline $40-49$ & 15 & 25 & 10 & 16.7 & \\
\hline $50-60$ & 30 & 50 & 42 & 70 & \\
\hline Mean \pm SD & $44.65 \pm 11.73$ & & $50.12 \pm 10.03$ & & $\begin{array}{l}\mathrm{t}=2.744 \\
\mathrm{P} 0.007^{*}\end{array}$ \\
\hline Gender & \multirow{2}{*}{21} & \multirow{2}{*}{35} & \multirow{2}{*}{36} & \multirow{2}{*}{60} & \multirow{3}{*}{$\begin{array}{c}\chi^{2}=7.519 \\
\text { P } 0.006^{*}\end{array}$} \\
\hline Male & & & & & \\
\hline Female & 39 & 65 & 24 & 40 & \\
\hline $\begin{array}{c}\text { Education } \\
\text { level }\end{array}$ & \multirow{2}{*}{21} & \multirow{2}{*}{35} & \multirow{2}{*}{29} & \multirow{2}{*}{48.3} & \multirow{4}{*}{$\begin{array}{c}\chi^{2}=2.219 \\
\text { P } 0.330\end{array}$} \\
\hline $\begin{array}{c}\text { Basic \& } \\
\text { below }\end{array}$ & & & & & \\
\hline Secondary & 27 & 45 & 22 & 36.7 & \\
\hline University & 12 & 20 & 9 & 15 & \\
\hline
\end{tabular}

Table 2 showed that more than half of the study group and slightly nearly half of the control group (51.7\% \& $45 \%)$ respectively had an ischemic stroke. More than two thirds of both the study group and the control group (66.7\% \& 63.3\%) respectively had referral admission from the medical ward. Regarding past medical history, nearly half $(45.3 \%)$ of the study group and more than half (56.7\%) of the control group respectively had neurological diseases. However, there were no statistically significant differences between the study group and the control group regarding health relevant data except diagnosis items $\left(X^{2}=11.614, p=0.035\right)$.
Table 2: Frequency and Percentage distribution of Health Relevant Data among the Study \& the Control Groups $(n=120)$.

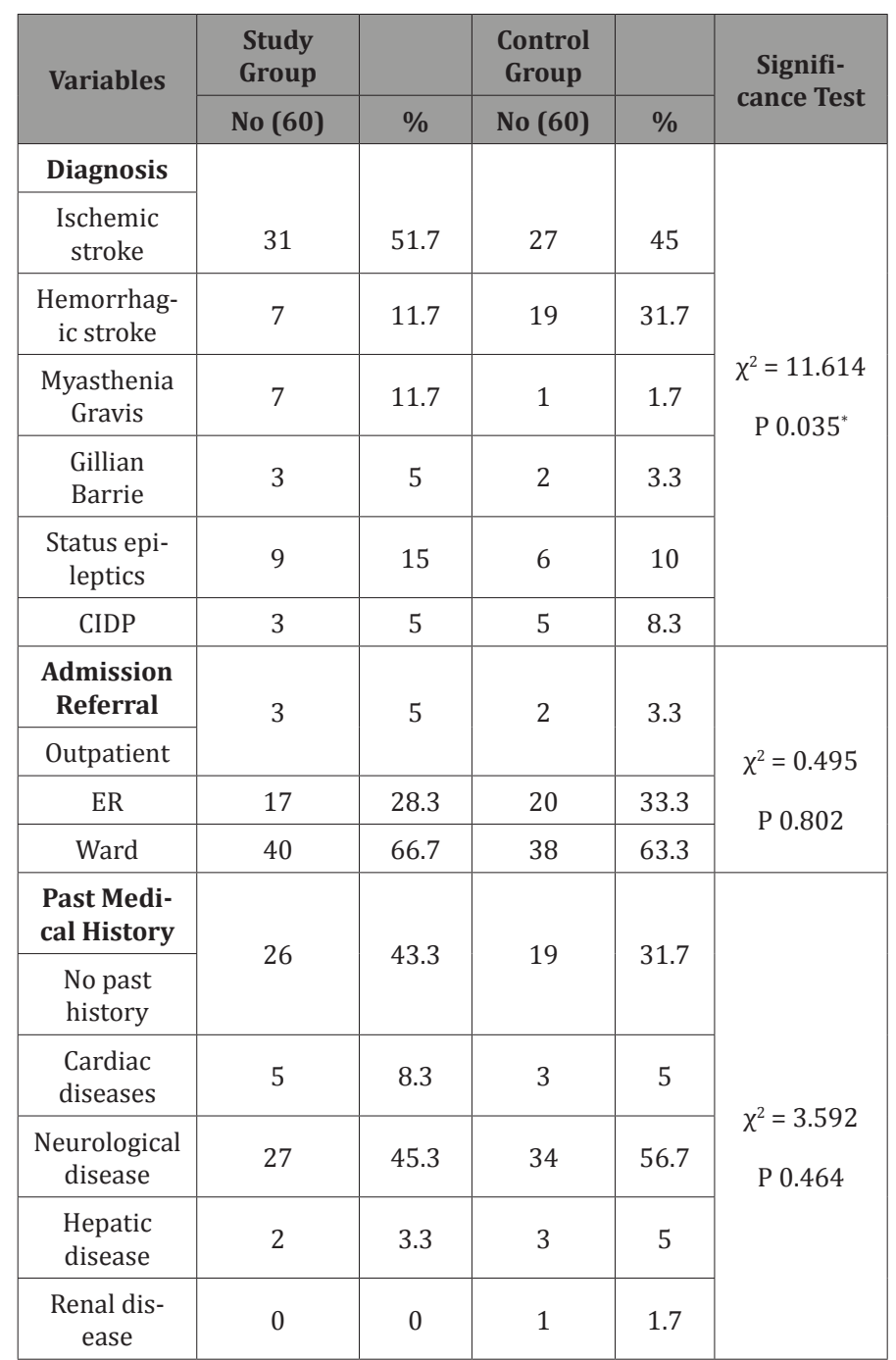

Table 3: Frequency and Percentage Distribution of patients demographic Data among The study \& The Control Groups $(n=120)$.

\begin{tabular}{|c|c|c|c|c|c|}
\hline \multirow{2}{*}{ Results } & \multicolumn{2}{|c|}{ Study Group } & \multicolumn{2}{|c|}{ Control Group } & \multirow{2}{*}{ Significance Test } \\
\hline & No (60) & $\%$ & No (60) & $\%$ & \\
\hline \multicolumn{5}{|c|}{ On Admission } & \multirow{4}{*}{$\begin{array}{c}\mathrm{X}^{2} 00.021= \\
\mathrm{p}=0.00^{0 *}\end{array}$} \\
\hline Nose & \multirow{2}{*}{0} & \multirow{2}{*}{0} & \multirow{2}{*}{0} & \multirow{2}{*}{0} & \\
\hline$+\mathrm{Ve}$ & & & & & \\
\hline -Ve & 60 & 100 & 60 & 100 & \\
\hline Groin & \multirow[b]{2}{*}{0} & \multirow[b]{2}{*}{0} & \multirow[b]{2}{*}{0} & \multirow[b]{2}{*}{0} & \\
\hline$+\mathrm{Ve}$ & & & & & \\
\hline$-\mathrm{Ve}$ & 60 & 100 & 60 & 100 & \\
\hline \multicolumn{5}{|c|}{$\begin{array}{l}\text { On Seventh } \\
\text { Day }\end{array}$} & \multirow{7}{*}{$\begin{array}{c}\mathrm{X}^{2} 00.021= \\
\text { p } 0.000^{*}\end{array}$} \\
\hline Nose & \multirow[b]{2}{*}{0} & \multirow[b]{2}{*}{0} & \multirow[b]{2}{*}{60} & \multirow[b]{2}{*}{100} & \\
\hline$+\mathrm{Ve}$ & & & & & \\
\hline$-\mathrm{Ve}$ & 60 & 100 & 0 & 0 & \\
\hline Groin & \multirow[b]{2}{*}{0} & \multirow[b]{2}{*}{0} & \multirow[b]{2}{*}{60} & \multirow[b]{2}{*}{100} & \\
\hline$+\mathrm{Ve}$ & & & & & \\
\hline$-\mathrm{Ve}$ & 60 & 100 & 0 & 0 & \\
\hline
\end{tabular}

As can be seen from Table 3 that no significant difference was found among both groups on admission. While the significant 
difference was found among both groups on the seventh day about swab from the nose $\left(X^{2}=120.00, P=0.000\right)$ and groin $\left(c^{2}=120.00, P=\right.$ 0.000 ) as the entire study group was free of MRSA while the entire control group was positive of MRSA.

\section{Discussion}

Body wash with tea tree oil has been successfully used as part of a decolonization strategy in many health care settings, but its specific benefits have not been delineated [22]. In our study, it was hypothesized that TTO body wash would reduce colonization of MRSA when compared to traditional body wash with soap and water. To our knowledge, the current research is the first study to evaluate the effectiveness of TTO body wash in preventing colonization with MRSA in critically ill patients in Egypt. The findings of this study showed that body wash of critically ill patients daily with a 5\% TTO among the study group had a highly significant reducing effect on the incidence of MRSA colonization when compared to traditional body wash with soluble soap and water among the control group.

This finding may have attributed to the better effect of tea tree oil on body wash as it has antibacterial, antifungal and antiseptic effects for the possible reasons as follows; the primary reason for TTO disrupts the vital function of the biological membrane. So, TTO treatment disturbed the MRSA membrane permeability, which allowed propidium iodide, a foreign substance, to enter the cell and potassium ions, part of the cytoplasmic material, to leave the cell [23]. Another possible explanation by other study has done by [24] which reported that TTO treated MRSA had increased susceptibility to sodium chloride $(\mathrm{NaCl})$, and become a cell toxin, and altered the cell membrane. Another possible factor that the TTO lotion wasn't rinsed and was kept till to the seventh day. As well, it improved bedside exposure to hospital routine care. This result is supported by [25] who conducted a randomized controlled trial of tea tree oil preparation for MRSA colonized wounds on 32 patients. 16 subjects in the control group received routine saline gauzed dressing, while the tea tree oil group patients received the $10 \%$ topical tea tree preparation dressing. That study revealed the $10 \%$ topical tea tree preparation was effective in reducing the quantity of colonized MRSA in and promoting healing of chronic wounds among the elderly.

Similarly, our study finding is consistent with [26] who showed that $99.9 \%$ of the MRSA was killed by TTO within 4 hours and that all MRSA was eradicated after 6 hours of continuous exposure to TTO 5\%. This finding suggested that TTO is a good agent for reduced transmission and control MRSA among humans.

On the other hand, Our study finding contradicted with a similar study done by [21] who conducted a study on tea tree oil (5\%) body wash compared with standard care (Johnson's baby soft wash) to prevent colonization MRSA on 391 patients, who were randomized to $(\mathrm{n}=196)$ patients received Johnson Baby Soft (JBS) body wash and195 patients received TTO 5\% body wash. That study found that washing of critically ill patients daily with a 5\% TTO body wash had no significant effect on the incidence of MRSA colonization in comparison with using JBS. The possible explanation of that finding that TTO body was applied and rinsed off the skin when compared to our study. Moreover, the current study contradicted with [27] who conducted a study on the randomized, controlled trial of used TTO based regimen (10\% nasal cream and $5 \%$ body wash) compared with standard topical treatment (nasal mupirocin 2\% and chlorhexidine 4\% body wash) for the clearance of MRSA colonization on 224 patients; as they found that no significant differences between the study group and the control groups regarding body wash. As well, this finding is inconsistent with [28] who reported that despite years of use, available clinical evidence does not support the effectiveness of tea tree oil to treat skin problems and human infections.

In addition, the researchers investigated the comparison of the study and control group by their demographic characteristics and found no significant differences were found between them except gender. Our finding is consistent with [21] who conducted a similar study and found no significant differences between study and control except gender. Moreover, the researchers noticed that old age in both groups was more susceptible to MRSA infection than other age groups due to a compromised immune response which is considered the risk factor that increases MRSA infection. Concerning health relevant data, the present study findings revealed [29] that there were no statistically significant between the study group and the control group. Our study finding coincides with the results of [21] who reported that more than one third of both groups had neurological diseases such as stroke who were susceptible to a prolonged hospital stay and more susceptible to get MRSA infection.

\section{Conclusion}

It can be concluded that all the study group of patients were free of MRSA while all the control of patients was positive of MRSA. Moreover, no significant differences were found between both groups regarding demographic characteristics except gender as well as health-relevant data.

\section{Acknowledgement}

Our sincere appreciation offered to all patients at Mansoura university Hospital who participating in this study. The authors wish to express their deepest gratitude to all people who shared or contributed in this valuable work.

\section{Conflict of Interest}

No Conflict of interest.

\section{References}

1. Moyo SJ, Nkinda L, Majigo M, Mmbaga E, Mbembati N, et al. (2017) Prevalence of methicillin-resistant Staphylococcus aureus carriage on admission among patients attending regional hospitals in Dar es Salaam, Tanzania. BMC research notes 10(1): 417.

2. Lee J, Austin JM, Kim J, Miralles PD, Kaafarani HM, et al. (2018) Developing and Testing a Chart Abstraction Tool for ICU Quality Measurement. American Journal of Medical Quality.

3. Kourtis AP (2019) Vital Signs: Epidemiology and Recent Trends in Methicillin-Resistant and in Methicillin-Susceptible Staphylococcus aureus Bloodstream Infections-United States. MMWR. Morbidity and Mortality Weekly Report 68(9): 214-219.

4. Xiao G, Chen Z, Lv X (2018) Chlorhexidine-based body washing for colonization and infection of methicillin-resistant Staphylococcus aureus and vancomycin-resistant Enterococcus: an updated metaanalysis. Infect Drug Resist 11: 1473-1481. 
5. Sabry NA, Farid SF, Dawoud DM (2014) Antibiotic dispensing in Egyptian community pharmacies: an observational study. Res Social Adm Pharm 10(1): 168-184.

6. Peres D, Pina E, Cardoso MF (2011) Methicillin-Resistant Staphylococcus Aureus (MRSA) in a Portuguese hospital and its risk perception by health care professionals. Revista Portuguesa De SaúdePública 29(2): 132-139.

7. Hanberger H, Walther S, Leone M, Barie PS, Rello J, et al. (2011) Increased mortality associated with meticillin-resistant Staphylococcus aureus (MRSA) infection in the Intensive Care Unit: results from the EPIC II study. Int J Antimicrob Agents 38(4): 331-335.

8. Centers for Disease Control and Prevention (US) (2013) Antibiotic resistance threats in the United States, 2013.Centers for Disease Control and Prevention, US Department of Health and Human Services.

9. Wong JG, Chen MI, Win MK, Ng PY, Chow A (2016) Length of stay an important mediator of hospital-acquired methicillin-resistant Staphylococcus aureus. Epidemiol Infect 144(6): 1248-1256.

10. Centers for Disease Control and Prevention )2015) Methicillin-resistant Staphylococcus aureus (MRSA) infections. CDC.

11. Vijayamohan N, Nair SP (2014) A study of the prevalence of methicillinresistant Staphylococcus aureus in dermatology inpatients. Indian dermatology online journal 5(4): 441-445.

12. Arias-Ortiz PM, Calderón LDP, Castillo JS, Moreno J, Leal AL, et al. (2016). Risk factors for methicillin-resistant Staphylococcus aureus bacteremia: A multicenter matched case-control study. Biomedica 36(4): 612-619.

13. Becker J, Diel R (2017) Screening for Methicillin-resistant Staphylococcus aureus in a residence home for elderly in Germany. J Occup Med Toxicol $12(1): 3$

14. Lowy FD (2015) Treatment of invasive methicillin-resistant Staphylococcus aureus infections in adults.

15. Kramlich D (2016) Strategies for Acute and Critical Care Nurses Implementing Complementary Therapies Requested by Patients and Their Families. Crit Care Nurse 36(6): 52-58.

16. Pazyar N, Yaghoobi R, Bagherani N \&Kazerouni A (2013) A review of applications of tea tree oil in dermatology. International Int J Dermatol 52(7): 784-790.

17. Baldissera MD, Souza CF, Doleski PH, De Vargas AC, Duarte MM, et al. (2017) Melaleucaalternifolia essential oil prevents alterations to purinergic enzymes and ameliorates the innate immune response in silver catfish infected with Aeromonashydrophila. Microbial pathogenesis 109: 61-66.
18. Kremsreiter A (2016) Tea Tree il as an Agent for MRSA Decolonization. Txicology 12(1): 3 .

19. Kramlich D (2014) Introduction to complementary, alternative, and traditional therapies. Crit care nurse 34(6): 50-56.

20. Ping LSK (2015) Role of complementary medicine in nursing and health care professionals. SOJ Nur Health Care 1(2): 1-2.

21. Blackwood B, Thompson G, McMullan R, Stevenson M, Riley TV, et al. (2013) Tea tree oil (5\%) body wash versus standard care (Johnson's Baby Softwash) to prevent colonization with methicillin-resistant Staphylococcus aureus in critically ill adults: a randomized controlled trial. Journal of Antimicrobial Chemotherapy 68(5): 1193-1199.

22. Bowler WA, Bresnahan J, Bradfish A et al.(2010) An integrated approach to methicillin-resistant Staphylococcus aureus control in a rural, regional-referral health care setting. Infect Control Hosp Epidemiol31(3): 269-275.

23. Cox SD, Mann CM, Markham JL, Bell HC, Gustafson JE, et al. (2000). The mode of antimicrobial action of the essential oil of Melaleucaalternifolia (tea tree oil). J Appl Microbiol 88(1): 170-175.

24. Carson CF, Mee BJ, Riley TV (2002) Mechanism of action of Melaleucaalternifolia (tea tree) oil on Staphylococcus aureus determined by time-kill, lysis, leakage, and salt tolerance assays and electron microscopy. Antimicrobial agents and chemotherapy 46(6): 1914-1920.

25. Lee RL, Leung PH, Wong TK (2014) A randomized controlled trial of topical tea tree preparation for MRSA colonized wounds. International Journal of Nursing Sciences.

26. May J, Chan CH, King A, Williams L, French GL (2000) Time-kill studies of tea tree oils on clinical isolates. Journal of Antimicrobial Chemotherapy 45(5): 639-643.

27. Dryden MS, Dailly S, Crouch M (2004) A randomized, controlled trial of tea tree topical preparations versus a standard topical regimen for the clearance of MRSA colonization. J Hosp Infect 56(4): 283-286.

28 Russell J, Rovere A (2009) “Tea Tree Oil”. American Cancer Society Complete Guide to Complementary and Alternative Cancer Therapies $\left(2^{\text {nd }}\right.$ edn), American Cancer Society, USA.

29 Berger B, Gumbinger C, Steiner T, Sykora M (2014) Epidemiologic features, risk factors, and outcome of sepsis in stroke patients treated on a neurologic intensive care unit. J Crit Care 29(2): 241-248. 